Trauma Berufskrankh 2016 - [Suppl 4]:

18:S340-S345

DOI 10.1007/s10039-016-0159-0

Online publiziert: 29. April 2016

๑) Springer-Verlag Berlin Heidelberg 2016

CrossMark
Christof A. Müller

Klinik für Unfall-, Hand- und orthopädische Chirurgie, Klinikum Karlsruhe, Karlsruhe, Deutschland

\section{Unterarm und Handgelenk} Komplexe Unterarmfrakturen
Die Therapie von Unterarmfrakturen setzt besondere Kenntnisse der komplexen Anatomie des Unterarmes voraus. Nur in Kenntnis der speziellen anatomischen und biomechanischen Verhältnisse ist es möglich, bei der Rekonstruktion von Frakturen und Bandverletzungen gute klinische und funktionelle Ergebnisse $\mathrm{zu}$ erzielen.

\section{Anatomie}

Definitionsgemäß besteht der Unterarm aus Radius und Ulna. Mithilfe einer gelenkigen Verbindung kann der Radius eine Umwendbewegung um die Ulna durchführen, die sog. Pro- und Supinationsbewegung. Voraussetzung für diese Umwendbewegung ist, dass der Radius eine Krümmung von ca. $10^{\circ}$ in beiden Ebenen aufweist und zudem eine Gelenkverbindung am proximalen und distalen Ende mit der Ulna besteht.

Am Unterarm gibt es somit 6 Gelenke:

- proximal:

- das Radiokubitalgelenk,

- das proximale Radioulnargelenk sowie

- das Ulnokubitalgelenk,

- distal:

- das Radiokarpalgelenk,

- das distale Radioulnargelenk sowie

- das Ulnokarpalgelenk.

Radius und Ulna werden zudem noch durch die Membrana interossea zusammengehalten. Somit ist der gesamte Unterarm eine funktionelle Einheit und als gelenkige Verbindung zu werten.

Das Ziel von Repositionen und Osteosynthesen am Unterarm ist, die anatomisch exakte Stellung wiederherzustellen. Es gelten somit am Unterarmschaft dieselben Regeln wie bei Gelenkrekon- struktionen. Ebenfalls ist die direkte Knochenbruchheilung - ohne oder mit nur wenig Kallusbildung - anzustreben, da eine große oder gar überschießende Kallusbildung zur Ausbildung eines Brückenkallus führen kann, der die Pround Supinationsbewegung einschränkt oder ganz unterbindet.

Als weitere Besonderheit ist der trianguläre fibrokartilaginäre Komplex (TFCC) zu nennen, der als knorpelige Scheibe wie ein Puffer zwischen Radius und Ulna am distalen Radioulnargelenk wirkt.

Bestehen bei der Umwendbewegung länger anhaltende Beschwerden, ist eine Verletzung des TFCCs mittels Magnetresonanztomographie (MRT) auszuschließen.

Einfache Unterarmfrakturen, wie am Beispiel einer 17-jährigen Reiterin dargestellt (•Abb. 1), werden standardmäßig mittels offener Reposition und nicht winkelstabiler Plattenosteosynthese versorgt („low contact dynamic compression plate“, LCDCP).

Bei dieser Verletzung finden die klassischen AO-Techniken (Arbeitsgemeinschaft für Osteosythesefragen) der offenen und anatomischen Reposition in Verbindung mit einer Kompressionsplattenosteosynthese Anwendung.

Insbesondere während der Facharztausbildung nimmt die Versorgung dieser Frakturen eine herausragende Stellung ein, da bei der osteosynthetischen Versorgung der einfachen Unterarmfrakturen die klassischen Osteosynthesetechniken gelehrt und gelernt werden können. Erzielt wird die interfragmentäre Kompression durch eine anatomische Reposition, das leichte Vorbiegen der Platte und nachfolgend das Einbringen der frakturnahen Schrauben im Plattenloch in frakturferner Position, wie dies bereits im ersten Manual der Osteosynthese beschrieben wurde [15]. Diese Techniken stellen die Basis einer jeden Ausbildung dar und müssen von jedem Facharzt sicher beherrscht werden.

Es stellt sich nun die Frage, warum man heute noch Techniken anwendet, die vor 50 Jahren entwickelt wurden?

Nach Aufkommen der winkelstabilen Plattenosteosynthese fand auch diese Technik am Unterarm Anwendung. Wie - Abb. 2 zeigt, kann auch mit einer „locking compression plate“ (LCP) eine interfragmentäre Kompression erreicht werden, indem die frakturnahen Schrauben - wie bei einer konventionellen Platte - nicht winkelstabil exzentrisch in Kompressionsstellung im Plattenloch eingebracht werden. Danach werden frakturferne Schrauben winkelstabil fixiert. Die winkelstabilen Schrauben führen dann nur noch zu einer Neutralisation der Kräfte.

Wissenschaftliche Untersuchungen zeigen, dass die klinischen und funktionellen Ergebnisse nach LCP-Osteosynthesen mit den Ergebnissen von konventionellen, nicht winkelstabilen Platten vergleichbar sind. Allerdings sind die Komplikationsraten bei der Metallentfernung von winkelstabilen Platten deutlich höher als bei der Implantatentfernung von konventionellen Platten, wie in $-\mathrm{Abb}$. 3a, b zu sehen. Daher ist es nicht notwendig, bei vergleichbarem klinischem Ergebnis die teureren winkelstabilen Implantate $\mathrm{zu}$ verwenden [7].

\section{Komplexe Unterarmfrakturen}

Bei komplexen Unterarmfrakturen handelt es sich einerseits um komplexe 

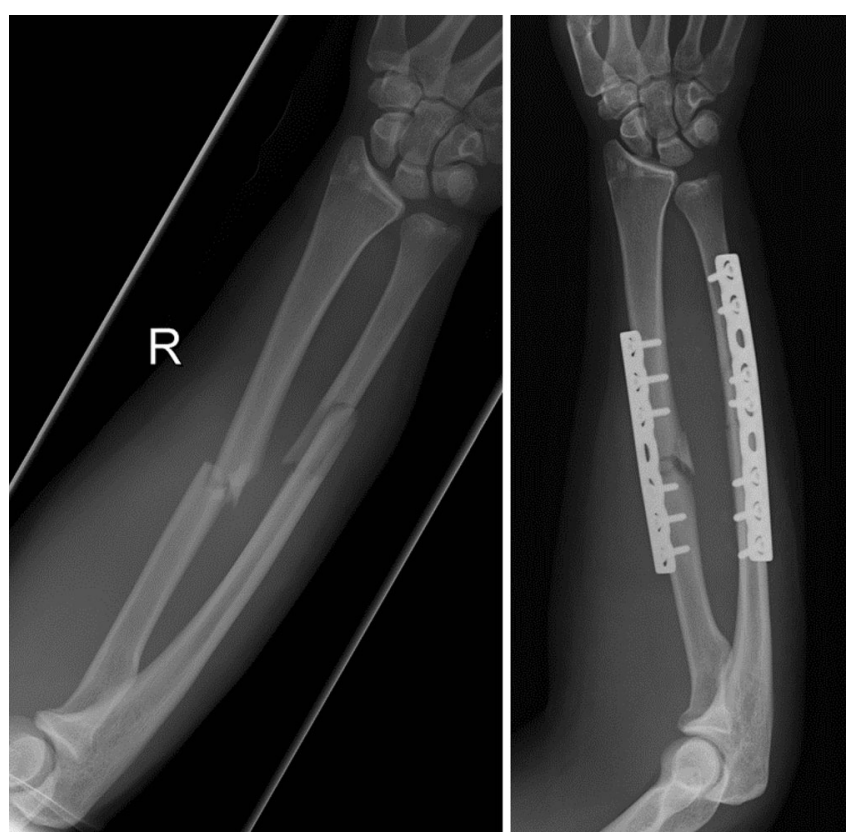

Abb. $1 \Delta$ a Einfache Unterarmfraktur:17-jährige Reiterin, die sich dieseFraktur nach Sturz vom Pferd zuzog. b Standardmäßige Versorgung mittels offener Reposition und nicht winkelstabiler Plattenosteosynthese („Iow contact dynamic compression plate“, LCDCP) mit interfragmentärer Kompression

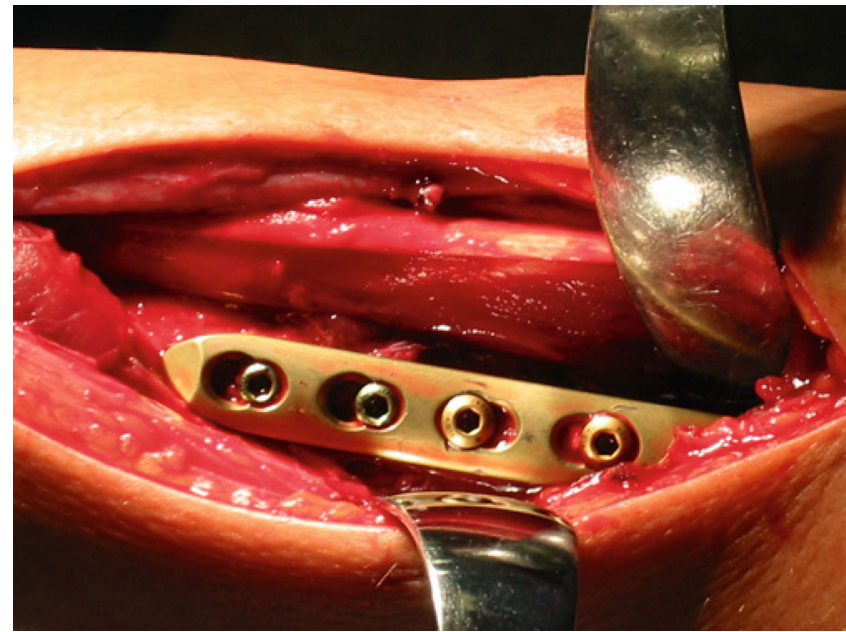

Abb. $2 \Delta$ Mittels winkelstabiler Plattenosteosynthese versorgte Unterarmfraktur. Das intraoperative Bild zeigt, dass sowohl nicht winkelstabile als auch winkelstabile Schrauben verwandt wurden. Mit den frakturnahen, nicht winkelstabilen Schrauben wurde zunächst die interfragmentäre Kompression durchgeführt. Mit den danach eingebrachten winkelstabilen Schrauben wird die Neutralisation der Kräfte durchgeführt
Frakturformen und andererseits um eine Kombinationsverletzungen von Fraktur und Gelenkluxation. Am Unterarm sind hier die Unterformen der Galeazzi-, Monteggia- und Essex-Lopresti-Verletzung zu nennen. Ebenfalls kann durch einen höheren Weichteilschaden und Defektfrakturen die Verletzungsschwere deutlich erhöht werden.

\section{Monteggia-Fraktur}

Von Monteggia-Frakturen oder Monteggia-Verletzungen spricht man, wenn es sich um eine proximale Ulnafraktur in Kombination mit einer Luxation des proximalen Radioulnar- und Humeroulnargelenkes - sog. Radiusköpfchenluxation - handelt.

Ein typischer Unfallmechanismus für diese Verletzung ist der Sturz auf den pronierten Unterarm oder ein direkter Schlag auf die Ulna. Dadurch kommt es zur Fraktur der proximalen Ulna mit begleitender Luxation des Radiusköpfchens.

Der Erstbeschreiber ist Giovanni Batista Monteggia (1792-1815), ein Mailänder Chirurg. Die Verletzung ist insgesamt selten und lediglich bei 2-5\% der Verletzungen des Unterarmes anzutreffen.

\section{Klassifikation nach Bado}

Monteggia-Frakturen werden nach Bado in 4 Typen eingeteilt (• Abb. $4[1,2]$ ). Der Typ 2 nach Bado wird seinerseits in 4 Subtypen nach Jupiter klassifiziert [9, 11]:

- Typ 1: Fraktur Ulnadiaphyse mit Angulation nach anterior, Luxation nach anterior,

- Typ 2: Proximale Ulnafraktur mit Angulation nach posterior,

1. Radiusköpfchen nach posterior,

2. mit Beteiligung des Processus coronoideus,

3. Ulnafraktur distal des Processus coronoideus,

4. Fraktur der Ulnadiaphyse mit Trümmerzone,

- Typ 3: Fraktur der Ulnametaphyse mit Angulation nach lateral und Luxation nach lateral,

- Typ 4: Ulna und Radiusfraktur mit Luxation nach anterior.

\section{Diagnostik}

Bei der radiologischen Diagnostik der Monteggia-Fraktur kommt es auf die genaue und korrekte Zuordnung der Gelenkstellungen an. Hier spielt insbesondere die Erfahrung des Betrachters eine große Rolle. Um die korrekte Gelenkstel- lung beurteilen zu können, muss die sog. Sören-Linie nachverfolgt werden. DieSören-Linie stellt die Verlängerung des Radiusschaftes in Bezug auf den Humerus dar. Hierbei muss das Zentrum der Linie in beiden Röntgenebenen exakt im Zentrum des Epicondylus humeri radialis stehen (• Abb. 5; [13]).

\section{Therapie}

Die Therapie der Monteggia-Fraktur ist, wann immer möglich, operativ. Es sollte eine frühzeitige Reposition durchgeführt werden, nachfolgend die anatomische Reposition und Osteosynthese der Ulna sowie die korrekte Reposition des Radiusköpfchens. Wichtig ist dabei, dass sowohl intraoperativ als auch postoperativ eine korrekte Reposition und Stellung des Radiusköpfchens vorliegen, da es teilweise zu sekundären Dislokationen im Behandlungsverlauf kommt. Durch Röntgenverlaufskontrollen muss die sekundäre Dislokation kontrolliert bzw. ausgeschlossen werden [16].

Monteggia-Frakturen können aber auch von weiteren Begleitverletzungen wie der Radiusköpfchen- und Coronoidfraktur begleitet sein, die ebenfalls therapeutisch angegangen werden müs- 
sen. Abhängig vom Frakturtyp erfolgt die Behandlung.

Bei der Radiusköpfchenfraktur Typ Mason I wird konservativ behandelt. Die Mason-II-Fraktur wird osteosynthetisch versorgt, und die Mason-III-Fraktur wird entweder, wenn möglich, osteosynthetisch oder mittels Radiusköpfchenprothese versorgt.

Insbesondere ist bei den Mason-TypIII-Frakturen auf die noch später beschriebene Essex-Lopresti-Verletzung zu achten.

Processus-Coronoidfrakturen werden nach Morrey eingeteilt: Die MorreyI-Verletzung wird konservativ, die Morrey-II-Verletzung mittels Ausziehnaht oder Schraubenosteosynthese und die Morrey-III-Verletzung mittels Schraubenosteosynthese versorgt.

Eine Sonderform stellt die „Monteggia like lesions" oder Monteggia-äquivalent-Verletzung dar. Darunter werden alle Monteggia-Frakturen mit Begleitverletzungen wie Radiusköpfchenfrakturen, Processus-Coronoidfrakturen oder Verletzungen der Kollateralbänder und der Membrana interossea subsumiert.

Da v. a. Begleitverletzungen die Prognose im wesentlichen Maß mit bestimmen, ist es wichtig, die Begleitverletzungen zunächst zu diagnostizieren, um sie nachfolgend in der Therapie mit berücksichtigen zu können.

\section{Galeazzi-Fraktur}

Bei der Galeazzi-Fraktur oder auch Galeazzi-Verletzung handelt es sich um eine Radiusfraktur in der Schaftmitte oder distal der Schaftmitte mit einer Luxation des distalen Radioulnargelenkes. Es kommt hierbei zu einer Zerreißung bzw. Teilzerreißung der Membrana interossea. Das klassische Unfallereignis ist der Sturz auf den supinierten Unterarm oder ein direkter Schlag auf den Radius. Diese Verletzung ist nach dem Erstbeschreiber, Ricardo Galeazzi (1866-1952), einem italienischen Chirurgen, benannt. Von allen Unterarmverletzungen ist diese Verletzung mit 3-7\% selten.

\section{Klassifikation}

Die Einteilung richtet sich nach der Reponierbarkeit $[3,10]$ :

Trauma Berufskrankh 2016 • [Suppl 4]: 18:S340-S345 DOI 10.1007/s10039-016-0159-0

(c) Springer-Verlag Berlin Heidelberg 2016

\section{A. Müller}

\section{Unterarm und Handgelenk. Komplexe Unterarmfrakturen}

\section{Zusammenfassung}

Bei der Versorgung von Unterarmfrakturen müssen die Prinzipien der anatomischen Reposition Anwendung finden. Der Unterarm ist in seiner Funktion als Gelenkverbindung zu sehen, und es werden nur gute klinische Ergebnisse erzielt, wenn die Prinzipien der Behandlung der Unterarmschaftfrakturen denjenigen von Gelenkfrakturen entsprechen. Bei Unterarmverletzungen ist immer auch auf Begleitverletzungen zu achten, die teilweise schwierig festzustellen sind. Insbesondere ist bei den Sonderformen wie Monteggia- und Galeazzi-Fraktur an typische Begleitverletzungen wie Coronoidund Radiusköpfchenfrakturen sowie die
Essex-Lopresti-Verletzung zu denken. Wenn möglich, sollte eine frühfunktionelle Behandlung angestrebt werden. Eine Ruhigstellung über mehr als 14 Tage kann zu Bewegungseinschränkungen führen. Bei den häufig vorliegenden Kombinationsverletzungen von Fraktur und Bandverletzung muss in der Nachbehandlung ein Kompromiss von zu langer und zu geringer Ruhigstellungszeit gefunden werden.

\section{Schlüsselwörter}

Galeazzi-Fraktur · Monteggia-Fraktur . Osteosynthese - Reposition - Therapie

\section{Forearm and wrist. Complex forearm fractures}

\section{Abstract}

In the treatment of forearm fractures the principles of anatomical repositioning must be applied. The function of the forearm must be considered as a joint connection and good clinical results can only be achieved when the principles of treatment of forearm fractures are comparable with those of joint fractures. In the diagnostics of forearm fractures the typical accompanying injuries must always be taken into consideration but which are sometimes difficult to determine. This particularly applies to the special forms, such as Monteggia and Galeazzi fractures with typical accompanying lesions, such as radial head or coronoid fractures as well as Essex-Lopresti lesions. When possible an early functional treatment should be strived for. Immobilization for more than 14 days can lead to impairment of mobility. For the commonly occurring combination injuries of fractures and ligament injuries, a compromise must be found in the follow-up treatment between too long and too short immobilization times.

\section{Keywords}

Osteosynthesis · Repositioning · Galeazzi fracture $\cdot$ Monteggia fracture - Therapy

$$
\begin{aligned}
& \text { - reponierbar und stabil, } \\
& \text { - reponierbar und instabil bzw. } \\
& \text { - nicht reponierbar. }
\end{aligned}
$$

\section{Diagnostik}

Wird eine Radiusschaftfraktur in der Schaftmitte oder distal der Schaftmitte festgestellt, muss eine klinische Untersuchung des distalen Radioulnargelenkes (DRUG) erfolgen. Besteht hier ein Druckschmerz, ist die Stabilität des DRUGs zu überprüfen, um eine Luxation oder Subluxationsstellung auszuschließen.

Somit ist es notwendig, dass eine subtile klinische Untersuchung erfolgt. Insbesondere nach erfolgter Osteosynthese ist intraoperativ die Stabilität des distalen
Radioulnargelenkes nochmals zu überprüfen.

\section{Therapie}

Die Therapie der Galeazzi-Fraktur ist stets operativ. Auch bei diesem Verletzungsmuster wird der Radiusschaft anatomisch reponiert und mittels stabiler Plattenosteosynthese versorgt.

Bei Mitverletzung des distalen $\mathrm{Ra}$ dioulnargelenkes und hier insbesondere des TFCC ist bei stabilen Verhältnissen eine funktionelle Therapie möglich. Bei Instabilität erfolgt entweder die offene Rekonstruktion und Naht des TFCC über einen Fadenanker, bei dynamischer Instabilität ist die Transfixation mit einem K-Draht und Ruhigstellung im Ober- 

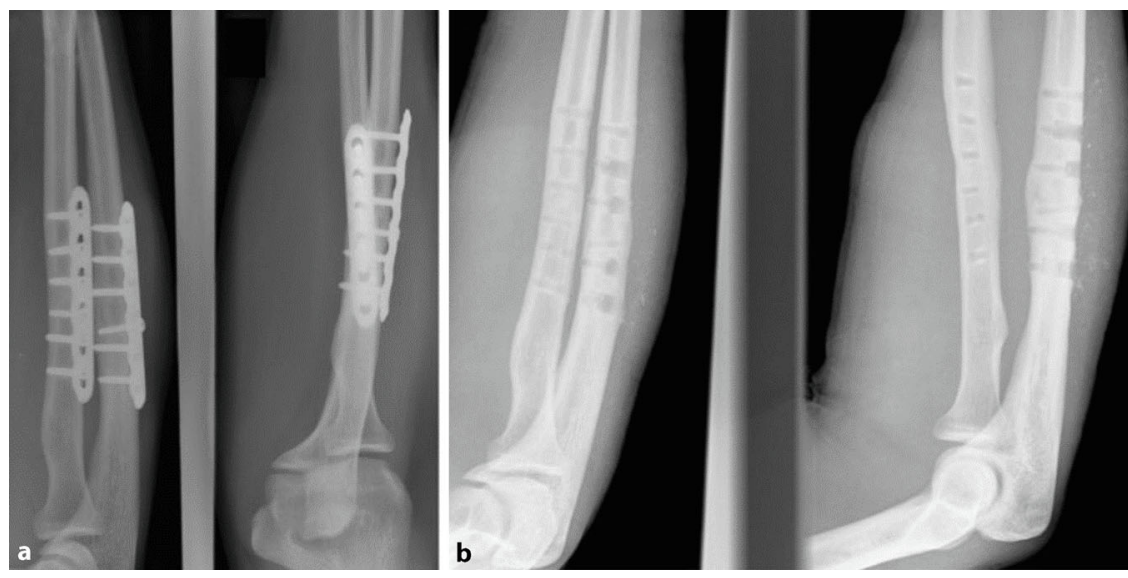

Abb. $3 \Delta$ a Gute knöcherne Konsolidierung. b Nach Implantatentfernung mit der Komplikation von festsitzenden Schrauben. Diese werden durch Überbohrung entfernt. Größere Knochendefekte, insbesondere an der Ulna, sind gut sichtbar. Durch diese Knochendefekte ist das Refrakturrisiko nach Implantatentfernung deutlich vergrößert. (Aus [7])

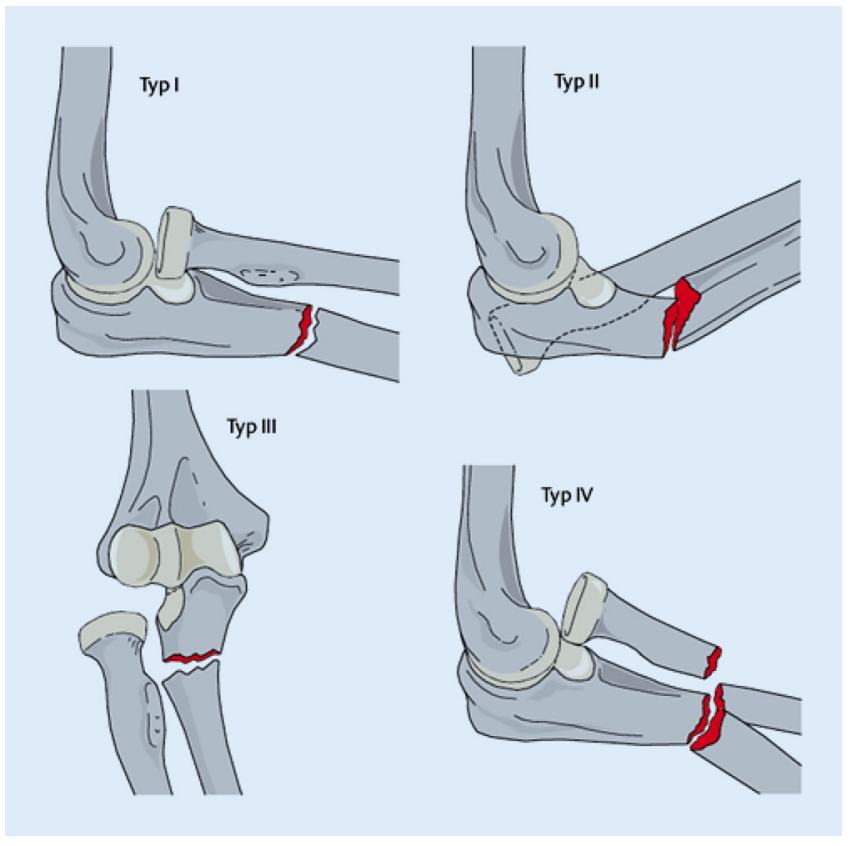

Abb. $4<$ Klassifikation nach Bado und Jupiter [1, 2, 9] (mit freundl. Genehmigung Georg Thieme Verlag [8])

armgips in Supinationsstellung notwendig. Die teilweise noch durchgeführte Stellschraubenosteosynthese sollte vermieden werden, da es durch die Schraube $\mathrm{zu}$ Knochendefekten und Verletzungen der Membrana interossea bzw. des TFCC kommen kann $[12,16,18]$.

\section{Kombinierte Monteggia- und Galeazzi-Läsionen}

Eine sehr seltene Verletzung ist auch die Kombination von Galeazzi- und Monteggia-Verletzung. Hierbei fällt zunächst die Unterarmfraktur auf. Wenn man allerdings das Röntgenbild näher analy- siert, fallen die Sprengung des distalen und die Luxation des proximalen Radioulnargelenkes ins Auge. Auch diese komplexe Verletzungskombination wird häufig primär übersehen und wird erst sekundär nach erfolgter Osteosynthese im weiteren Behandlungsverlauf durch Luxationen bzw. Subluxationen der Radioulnargelenke auffällig.

Daher ist es bei diesen komplexen Verletzungen wichtig, neben der anatomischen Rekonstruktion von Radius und Ulna auf die Stabilität im proximalen oder distalen Radioulnargelenkzu achten und bei Instabilität entsprechend operativ bzw. konservativ vorzugehen [14].

\section{Essex-Lopresti-Verletzung}

Die Essex-Lopresti-Verletzung ist eine seltene Verletzung, die häufig übersehen wird und erst sekundär - bei Auftreten von Komplikationen - auffällt. Es handelt sich um eine Radiusköpfchenfraktur mit Verletzung der Membrana interossea. Wenn die Membranzerreißung bis nach distal verläuft, kann es zur Mitverletzung des distalen Radioulnargelenkes kommen. In diesen Fällen führt die Instabilität zur Dislokation der Ulna nach distal, dem sog. Shiften. Ein weiterer Mechanismus, der das Shiften der Ulna begünstigt, ist die Verkürzung des Radius aufgrund einer komplexen Radiusköpfchenfraktur. Wegen der frakturbedingten Radiusverkürzung und der Instabilität aufgrund der Membranverletzung kommt es zum Distalshift der Ulna mit der Folge des ulnokarpalen Impingements.

Die Folge von nicht oder ungenügend behandelten Essex-Lopresti-Verletzungen ist das radiohumerale Impingement durch eine Dislokation des Radius nach proximal. Dadurch bedingt, kommt es zum relativen Ulnavorschub und zum ulnokarpalen Impingement (• Abb. 6; [5, 16]).

\section{Klassifikation}

Die Essex-Lopresti-Verletzung wird nach Jupiter in 3 Typen klassifiziert:

- Typ I: wenige große dislozierte

Radiusköpfchenfragmente,

- Typ II: erhebliche Fragmentation des Radiusköpfchens,

- Typ III: ältere Verletzung mit irreversibler Proximalmigration des Radius.

\section{Diagnostik}

Die Diagnostikstellt sich häufig schwierig dar. Eine Voraussetzung für die korrekte Diagnosestellung ist zunächst, dass der Untersucher die Essex-Lopresti-Verletzung kennt und nach ihr fahndet. Mit einer intraoperativen dynamischen Untersuchung unter Bildverstärker kann man das Shiften des Radius gegenüber der Ulna feststellen. Auch bildgebende Verfahren wie die Sonographie oder MRT-Untersuchungen stehen zur Diagnosestellung zur Verfügung. Da es schwierig ist, 

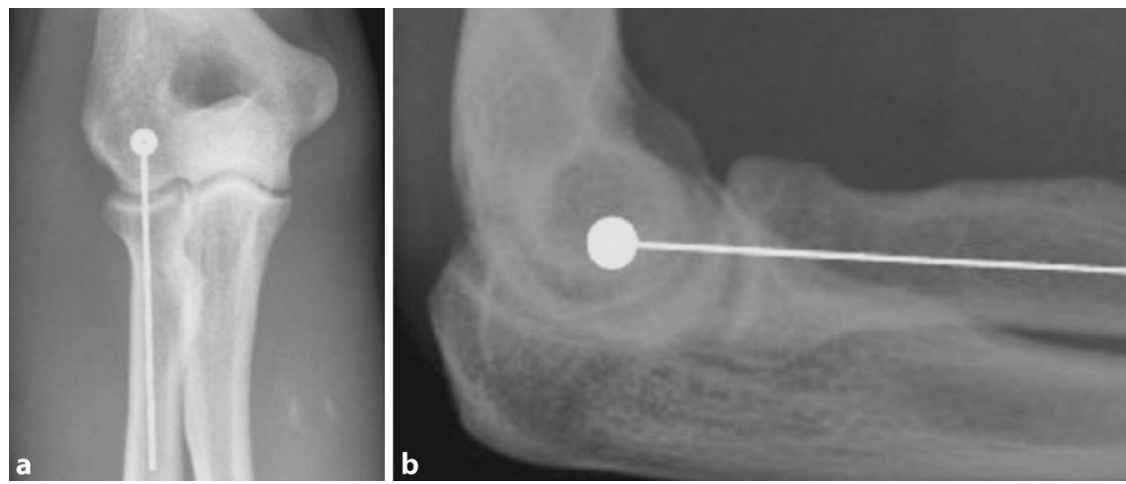

Abb. 5a, b \ Sören-Linie [13]

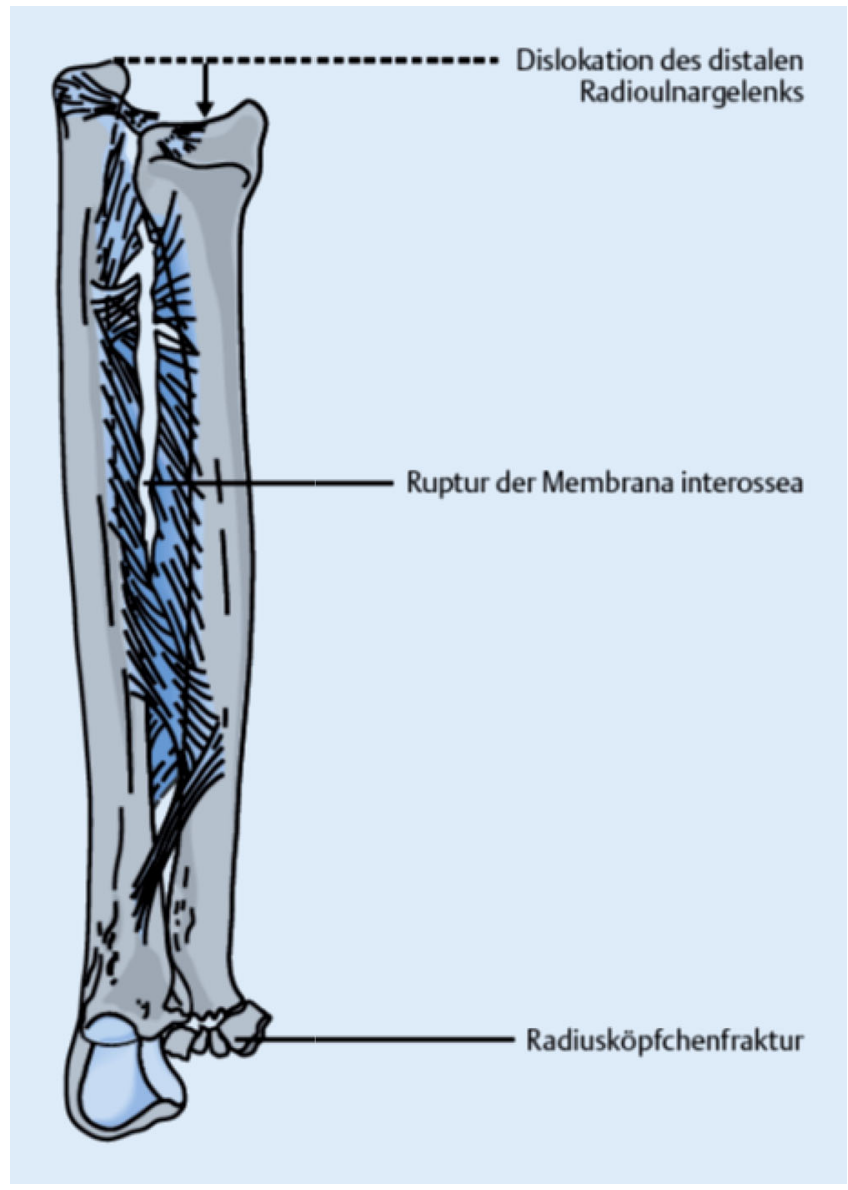

Abb. $6<$ Essex-Lopresti-Verletzung [19] (mit freundl. Genehmigung Georg Thieme Verlag)

diese Verletzung sicher zu diagnostizieren, wird angenommen, dass ungefähr ein Viertel der Verletzungen übersehen und erst sekundär entdeckt wird $[5,17]$.

\section{Therapie}

Bei Vorliegen einer Essex-Lopresti-Verletzung sind die Implantation einer Radiusköpfchenprothese und ggf. Osteosynthese, sofern möglich, Mittel der Wahl, um stabile Verhältnisse und eine korrekte Länge des Radius zu erhalten.
Bei sekundär festgestellter Verletzung mit den Spätfolgen des ulnokarpalen Impingements ist die Ulnaverkürzungsosteotomie eine mögliche Korrekturform.

\section{Weichteilschaden und Defektfrakturen}

Weitere komplizierende Faktoren der Unterarmfraktur sind Weichteilprobleme durch Schwellung und Kompartmentsyndrom. Bei diesen Situationen sollten frühzeitig eine Fixateur-externeRuhigstellung und ggf. auch eine Kompartmentspaltung Anwendung finden.

Frakturheilungsprobleme, die durch höhergradige Weichteilschäden begünstigt werden, verlängern ebenfalls deutlich den Behandlungszeitraum.

Die Bildung von Brückenkallung mit Einsteifung des Unterarmes oder weiter bestehende Instabilitäten des proximalen oder distalen Radioulnargelenkes führen ebenfalls zu schlechten Ergebnissen mit sehr langen Behandlungsverläufen.

Ein weiteres Problem stellen Defektfrakturen dar. Die Rekonstruktion von kleineren Defektstrecken kann mit der Auffüllung von Beckenkammspongiosa erfolgen. Bei mehreren Zentimeter großen Defekten kommen Verfahren der mikrovaskulären Knochentransplantation oder die neue Methode nach Masquelet in Kombination mit einem RIA (Reamer/Irrigator/Aspirator)-Graft zur Anwendung [6].

\section{Ergebnisse}

Klinische Nachuntersuchungen zeigen, dass bei verletzungstypgerechter Therapie Monteggia-Frakturen des Erwachsenen meist mit guten und sehr guten Ergebnissen ausheilen. Die Ergebnisse selbst sind allerdings stark vom Frakturtyp abhängig.

Bei Monteggia-Frakturen des Typ I, III und IV ergeben bei möglichst kurzer Ruhigstellungszeit sehr gute und gute Ergebnisse. Voraussetzung ist allerdings eine frühfunktionelle Behandlung. Die Ruhigstellungszeit sollte dabei 14 Tage nicht überschreiten.

Der Typ IIa ist allerdings ein Sonderfall. Bei diesem Verletzungstyp sind lediglich überwiegend nur befriedigende und schlechte Ergebnisse zu erzielen [4, 10].

\section{Fazit für die Praxis}

- Bei der Versorgung von Unterarmfrakturen müssen die Prinzipien der anatomischen Reposition Anwendung finden.

- Bei Unterarmverletzungen ist immer auf Begleitverletzungen zu achten. 


\section{- Bei den Sonderformen wie Mon- teggia- und Galeazzi-Fraktur muss an typische Begleitverletzungen wie Coronoid- und Radiusköpfchen- frakturen sowie die Essex-Lopresti- Verletzung gedacht werden. \\ - Eine frühfunktionelle Behandlung sollte angestrebt werden. Bei Kom- binationsverletzungen von Fraktur und Bandverletzung muss in der Nachbehandlung ein Kompromiss zwischen zu langer und zu geringer Ruhigstellungszeit gefunden werden.}

\section{Korrespondenzadresse}

\section{A. Müller}

Klinik für Unfall-, Hand- und orthopädische Chirurgie, Klinikum Karlsruhe

Moltkestr. 90, 76133 Karlsruhe, Deutschland ortho-trauma@klinikum-karlsruhe.de

\section{Einhaltung ethischer Richtlinien}

Interessenkonflikt. C.A. Müller gibt an, dass kein Interessenkonflikt besteht.

Dieser Beitrag beinhaltet keine vom Autor durchgeführten Studien an Menschen oder Tieren.

The supplement containing this article is not sponsored by industry.

\section{Literatur}

1. Bado IL (1958) La lesion de Monteggia. InterMedica Sarandi. SRL, , S328-330

2. Bado IL (1967) The Monteggia lesion. Clin Orthop 50:71-86

3. Clare DJ, Corley FG, Wirth MA (2002) Ipsilateral combination monteggia and galeazzi injuries in an adult patient: a case report. J Orthop Trauma 16(2):130-134

4. Conrad GG, Kundel K, Kreuz PC, Oberst M, Suedkamp NP (2007) Monteggia fractures in adults: long-term results and prognostic factores. J Bone Jt Surg 89-B:354-360

5. Edward GS, Jupiter JB (1988) Radial head fractures with acute distal radioulnar dislocation; EssexLopresti revisited. Clin Orthop Rel Res 234:61-69

6. Hanna W, Mueller CA (2012) RIA grafting for the treatment of a large bone defect in the distal radius - First case report of a new treatment option. Injury 44(2):19-22. doi:10.1016/j.injury.2012.10.006

7. Henle P, Ortlieb K, Kuminack K, Mueller CA, Suedkamp NP (2011) Problems of bridging plate fixation for the treatment of forearm shaft fractures with the locking compression plate. Arch Orthop Trauma Search 131(1):85-91

8. Josten C, MarquaßB (2006) Frakturen, Luxationen, Bandrupturen - Ellbogen, Unterarm. Orthopädie und Unfallchirurgie up2date 1:447-476
9. Jupiter JB, Leibovic SJ, Ribbans W, Wilk R (1991) The posterior monteggia lesion. J Orthop Trauma 5:395-402

10. Jupiter JB, Kellum JF (1998) Diaphyseal fractures of forearm. In: Browner BD, Jupiter JB, Legvine AM (Hrsg) Skeletal trauma. Saunders, Philadelphia

11. Korner J, Hoffmann A, Rudig L et al (2004) Monteggia-Verletzungen im Erwachsenenalter. Unfallchirurg 107:1026-1040

12. Lechner J, Steiger R, Ochsner P (1993) Die Operative Behandlung der Galeazzi-Fraktur. Unfallchirurg 96:18-23

13. Lendemans, Taeger G, Nast-Kolb (2008) Luxationsfraktur des Unterarms, Galeazzi, Monteggia und Essex-Lopresti-Verletzung. Unfallchirurg 111:1005-1016

14. Letta C, Schmied M, Haller A, Rindlisbacher A (2012) Kombinierte Monteggia und Galeazzi-Läsionen des Unterarmes. Unfallchirurg 115:1034-1037

15. Müller ME, Allgöwer M, Schneider R, Willenegger R (1969) Manual der Osteosynthese. Springer, Heidelberg

16. Reckling FW (1982) Unstable fracture-dislocations oft he forearm (Monteggia and Galeazzi lesion). J Bone Jt Surg A 64:857-863

17. Ries C, Wegmann K, Hackl M, Burkhart KJ, Müller LP (2016) Essex-Lpresti-Verletzung - doch nicht so selten? Orthop Unfallchir Prax 3:154-159

18. Rothe M, Rudy T, Stankovic P, Stürmer KM (2001) Die Therapie der Galeazzi-Fraktur - ist die Revision des distalen Radioulnargelenkes zwingend notwendig? Handchir Mikrochir Plast Chir 33(4):252-257

19. Wirth CH, Zichner LS (2005) Orthopädie und Orthopädische Chirurgie. In: Martini AK (Hrsg) Ellbogen, Unterarm, Hand. Georg Thieme Verlag, Stuttgart 